\title{
Un Ortega sin Weimar: tecnología y entendimiento agente en "La idea de principio en Leibniz"
}

\author{
Ignacio Quintanilla Navarro, Escuela de Filosofía de Madrid, España
}

Resumen: Ortega apenas habla de la técnica entre 1935 y 1951. Este silencio se analiza desde "La idea de Principio en Leibniz". La conclusión es que Ortega regresa a una interpretación productivo-pragmática de la ejecutividad la vida humana -como la propuesta en la "Meditación de la técnica"-cuando necesita marcar distancias con Heidegger. Este desarrollo argumental, abandonado en los años 30, se retoma en los 50 con un proyecto, nuevamente frustrado, de ontología modal en el que la pregunta por la posibilidad del ser aparece como previa a la pregunta por el ser mismo. Este trabajo trata de reconstruir la argumentación que Ortega no completa y de justificar el valor ejemplar que posee este episodio para entender el estado actual de la filosofia de la técnica.

Palabras clave: Filosofía de la técnica, historia de la filosofía de la técnica, ontología modal, Ortega y Gasset, Leibniz, optimismo filosófico, lógica de los mundos posibles

Abstract: Between 1935 and 1951 Ortega wrote almost nothing about technology. This silence is analized form the point of view of "La Idea de Principio en Leibniz". The conclusion is that Ortega returns to a productive-pragmatic interpretation of the human live as ejecution-like the expressed in the "Meditación de la técnica"- when he needs to take distance from Heidegger. This argumental line, forgotten in the 30s, is taken again in the 50s, with a project of modal ontology, also frustrated. In this project, the question for the possibility of the being appears more radical than the question for the being himself. This work tries to reconstruct the argumentation that Ortega does not complete and to justify the exemplary value that has this event in order to understand the situation of the philosophy of technology at the present.

Keywords: Philosophy of Technology, History of the Philosophy of Technology, Ontology, Modal Ontology, Ortega y Gasset, Leibniz, Philosophical Optimism, Logic of the Possible Worlds

\section{El enigma de la técnica en Ortega}

$\mathrm{H}$ ABLANDO EN CIERTA ocasión en Alemania, Ortega declaraba con modestia que él no era, en absoluto, un especialista en Goethe, un Goethe-forscher. Dicho lo cual, y sin ningún rebozo, pasaba a vindicar su interpretación de Goethe como la única verdaderamente atinada y a denunciar un error de enfoque básico en todos los Goethe-forscher alemanes, alguno de los cuales, posiblemente, le escuchaba. Y es que no solían faltarle a don José, como es sabido, redaños argumentales para liarse cualquier manta a la cabeza. Ahí está, si no, ese otro célebre comienzo de conferencia -en este caso madrileña- cuando señalando a José María Cossío, allí presente, dice y escribe: “... estoy seguro que él, más que nadie y con más fundamentos que nadie, está dispuesto a reconocer que si hay alguien en el mundo que sepa lo que es el toreo - esa bicentenaria realidad histórica española- ese alguien resulto ser yo"1. En agosto del 51 Martin Heidegger se beneficiaría de

1. Una interpretación de la historia universal, Cap. VII. (IX. 1298). Las referencias a la obra de Ortega se remiten

Revista Internacional de Tecnología, Ciencia y Sociedad

Volumen 2, Número 1, <http://tecnociencia-sociedad.com>, ISSN 2530-4895

(C) Global Knowledge Academics. Ignacio Quintanilla Navarro. Todos los Derechos Reservados

Permisos: soporte@gkacademics.com

Republicado de Revista Internacional de Tecnología, Conocimiento y Sociedad 2(1), 2013 (pp. 1-18) 
esta misma audacia argumental en el célebre congreso de Darmstadt. Allí, y pese a la radical diferencia de planteamientos constatada, no dudó nuestro filósofo en echar una mano al alemán, que se veía apurado con uno de los participantes -un gran arquitecto, dirá Ortega refiriéndose a este mismo incidente. Heidegger recordará también este episodio al esbozar cinco años después una semblanza laudatoria de Ortega. En esta semblanza alude también a un último encuentro entre ambos en el que Ortega le sorprendió al tratar el concepto de ser con cierta "especie de positivismo" sobre el que Heidegger dice que "no le corresponde juzgar"2.

Traigo a colación estas referencias por partida triple. En primer lugar porque debo yo también ahora comenzar por declarar que no soy un verdadero Ortega-forscher, aunque su filosofía de la técnica me ha cautivado y ocupado desde hace ya muchos años, lo que no me va a impedir meterme en las camisas de once varas que seguidamente se verán, en el más puro estilo del maestro. En segundo lugar porque el argumento que Ortega defiende sobre Goethe en la primera ocasión señalada - una conferencia pronunciada con distintas variantes en Aspen, primero, y en Hamburgo, Berlín y Stuttgart después y que lleva por título: Un Goethe sin Weimar-, guarda un hondo paralelismo con el que quiero yo defender aquí sobre el propio Ortega a propósito de su filosofía de la técnica. Finalmente, porque sobre las cuitas de Ortega con el ser en los últimos años de su vida, y esos titubeos ontológicos que a Heidegger le parecieran sorprendentes y con un matiz algo "positivista", esperamos arrojar también aquí alguna luz. Aunque, en este caso, se trate de mostrar una aventura intelectual ante la que Ortega se echó atrás.

Nuestro enfoque es simple. Ortega es el primer filósofo profesional de talla que elabora y publica una filosofía de la técnica como tal. Se trata, además, de una doctrina consistente, sugestiva y vigorosa, publicada por entregas el año 35 en el diario La Nación $\square$ GHBuenos Aires y como libro con el título Meditación de la técnica en el año 1939, es decir, significativamente antes de la Segunda Guerra Mundial, que es el evento que espabila la conciencia tecnológica de la mayoría de los filósofos europeos, con Heidegger y los frankfurtianos a la cabeza. Pero junto a este hecho convive otro no menos llamativo: parece innegable que tras el año 35 Ortega sigue elaborando su obra como si se hubiera olvidado de la Meditación -salvo para publicarla en el 39 de manera, como sabemos, un tanto forzada. En realidad, las referencias posteriores a la misma dan la impresión de que Ortega tiene sus reservas a la hora de otorgar a su filosofía de la técnica una validez definitiva. Y así, mientras en la obra de un Heidegger o un Marcuse - por citar ilustres nacidos en la última veintena del XIX-la técnica se va perfilando como un motivo argumental y editorial de creciente importancia desde los años cuarenta, en Ortega se va agotando en unas pocas, breves y no siempre coherentes observaciones incidentales.

Es verdad que este olvido se disimula bien por el empleo abundante, aunque siempre limitado y ad hoc, del conocido tema del ensimismamiento, el extrañamiento y la fantasía como origen de la condición humana -ese divino descontento que se ha denominado a veces

a la última edición de las obras completas, realizada por la Fundación Ortega y la editorial Taurus en Madrid, en diez volúmenes entre 2004 y 2010.

2. Begegnungen mit Ortega y Gasset (1955), en Martin Heidegger Gesamtausgabe, Band 13 Aus der Erfahrung des Denkens, Sch.17, Frankfurt del Main, V. Klostermann, 1983. 
racionalismo fantástico. Es verdad, también, que el período comprendido entre el 36 y el 50 no es un buen momento para tecnófilos, ni en Europa ni en España, y queda ya muy atrás ese soplo regeneracionista que en 1918 hacía escribir a un Lorca joven, a propósito de las viejas ciudades castellanas: "y sobre las rojizas cruces veremos un aeroplano triunfante"3. Se verían pronto, es verdad, muchos aeroplanos sobre los campanarios de Europa, pero no eran ése.

No obstante, Ortega no fue nunca tan tecnófilo como para no dejar abiertas, ya en la propia Meditación, dignas retiradas argumentales que la peripecia europea le permitía explotar. Además, como es notorio, La rebelión de las masas -la obra de Ortega que más se contradice con la Meditación pese a gestarse virtualmente en paralelo- colocó pronto a don José, de manera vitalicia, a cubierto de cualquier reproche de ceguera histórica. No basta, pues, con el contexto histórico para aclarar nuestro problema.

\section{Filosofía de la técnica: un estado de la cuestión}

La presente interpretación no se centra tanto en la propia Meditación de la técnica, el primer gran enigma en la producción de Ortega, cuanto en ese segundo gran enigma de su producción que es La idea de Principio en Leibniz, en adelante IPL. Nuestra hipótesis es que los vínculos argumentales entre la filosofía de la técnica orteguiana y la $I P L$ permiten esclarecer el problema fundamental que para Ortega suponía su Meditación. Sin embargo, nuestra intención es todavía un poco más ambiciosa, y apunta más allá de la mera exégesis orteguiana, pues intentaremos mostrar cómo el destino de la filosofía de la técnica en la obra de Ortega prefigura en buena medida el destino de toda la filosofía de la técnica en el contexto de la filosofía actual.

Ortega no fue capaz, o no quiso, llevar hasta las últimas consecuencias la teoría de la técnica que se contiene en la Meditación, y en esta deserción se esconde, también, una clave para comprender el estatuto de la filosofía de la técnica hoy. Su figura como primer filósofo profesional de la técnica adquiere de este modo un nuevo valor emblemático, y así como Ortega interpreta las "deserciones" vitales de Goethe -y la más flagrante de ellas es su prolongada instalación y remoloneo en Weimar- como un hito para entender la encrucijada de la cultura europea, así también la "traición" de Ortega al proyecto argumental que encierra su filosofía de la técnica -y su feliz y prolongado remoloneo en el mero nivel de la razón histórica- posee, a nuestro juicio, un valor paradigmático y actual.

En efecto, la filosofía de la técnica es la gran tarea del filósofo hoy y ha sido ya -hoy lo sabemos- el gran tema oculto de todo el pensamiento del siglo XX; aunque la mayor parte de sus protagonistas no fueran conscientes -o plenamente conscientes- de ello. En la reflexión sobre la técnica, más o menos incidental o explícita, encontramos el único común denominador en ese Babel de corrientes, lenguajes y enfoques que es la filosofía del siglo XX de Husserl a Wittgenstein, de Heidegger a Dewey, de Benjamin a Gadamer. A partir de esta constatación, la Meditación de la técnica accede al rango de obra emblemática en la filosofía del siglo XX, muy por encima de cualquier otra de las escritas por Ortega.

\footnotetext{
3. "Meditación” en Impresiones y paisajes, 1918.
} 
Y, sin embargo, el hecho es que la filosofía de la técnica sigue sin ser una disciplina sustantiva en el contexto de nuestra cultura e incluso en el propio cuerpo filosófico ${ }^{4}$. Naturalmente, perfilar el alcance de este diagnóstico exige alguna matización. Por lo pronto, ni siquiera en la propia Meditación se vindica seriamente una filosofía de la técnica como disciplina nueva dentro de la filosofía, en pie de igualdad con la ética o la teoría del conocimiento. No debe darse por sentado, pues, que promover una disciplina académica explícita y pujante sea la batalla crucial para quienes creemos que la filosofía de la técnica es el tema de nuestro tiempo ${ }^{5}$. Pero además sucede que esta deficiencia académica y cultural es perfectamente compatible con el hecho de que, a fecha de hoy, la filosofía de la técnica dispone de un corpus bibliográfico de tal volumen y calidad que justifica la apelación a algunos autores como clásicos así como la solidez de una literatura de segundo nivel consagrada a su crítica y sistematización. Como en este mismo congreso participan algunos de esos clásicos, no parece preciso dar nombres ahora.

Apoyándonos en esta sólida tradición estamos perfectamente capacitados para recapacitar sobre nuestro punto de partida y preguntar abiertamente: ¿por qué es tan importante la técnica para la filosofía? Podemos presentar el balance de casi un siglo de meditación filosófica sobre la técnica respondiendo a esta cuestión con tres afirmaciones: 1) porque si la sociedad demanda hoy filosofía es, sobre todo, para comprender y orientar su cambio tecnológico, 2) porque los principales problemas éticos y epistemológicos de nuestro tiempo se dilucidan teóricamente como opciones tecnológicas concretas, y 3) porque la técnica es

\footnotetext{
4. Sólo muy tardíamente la UNESCO ha recogido la filosofía de la técnica como una subdisciplina de la filosofía social -la 720703- al nivel del materialismo dialéctico o la teoría de las ideologías; nuestras universidades siguen sin tener departamentos de filosofía de la técnica, y -sin duda lo más grave-la mayor parte de los trabajos filosóficos que se publican sobre el tema, cada vez menos, pero todavía, son incursiones en un "tema de actualidad" desde modelos filosóficos y enfoques que ya se tienen pensados de antemano en contextos argumentales distintos de la propia técnica. En este sentido no deja de ser muy ilustrativo el minúsculo papel que, hasta hoy, la exégesis orteguiana ha solido otorgar a la filosofía de la técnica. Cuando el propio Garagorri edita en el 82 la Meditación y la completa, naturalmente, con El mito del hombre allende la técnica del 51 y En torno al coloquio de Darmstadt del 53, estos últimos escritos se presentan en un epígrafe titulado: Otros ensayos sobre ciencia y filosofía que incorpora, además -y como justificación editorial- otros dos trabajos de los años treinta sobre física. Por lo demás, el primer artículo publicado sobre el tema de la técnica en Ortega data de 1960, cuando Juan Vaya Menéndez publica "La cuestión de la técnica en una doble meditación: Ortega y Heidegger." Convivium, n 9-10, 1960, pp. 69-91. El último -excluido el del profesor Mermall que se contiene en este volumen- es el de González Quirós de 2006- referido en este trabajo. Entre medias hay, según los datos de la Fundación Ortega, y junto a tres interesantes trabajos de profesores italianos -uno de ellos tesis doctoral-, quince trabajos más en castellano, de muy variada índole y extensión y que sólo en menos de la mitad de los casos de consagran específicamente y en profundidad a la cuestión de la técnica en Ortega. Si a esto añadimos la inestimable labor de traducción y difusión realizada por el profesor Mitcham en los Estados Unidos, así como labor de los profesores Mermall y Patrick M. Dust en ese mismo país, y alguna que otra referencia incidental en Francia y en Alemania, (B $\square$ Stiegler y F. Rapp). Tenemos una idea bastante aproximada del estado de los estudios sobre la filosofía de la técnica orteguiana hasta la celebración del congreso de Madrid del 2010.

5. La propuesta neopragmatista del profesor Durbin de concebir al filósofo como una especie de "asistente social de las ideas" que trabaja sobre el terreno y justifica su responsabilidad social mediante la implicación personal en la resolución de problemas concretos, me parece, en este sentido, muy lúcida y constituye un reto para la filosofía de la técnica hoy. Cf. "Pragmatismo y tecnología”, Isegoría 12 (1995).
} 
necesaria para comprender lo que la filosofía occidental ha sido y es, es decir, porque de una filosofía de la técnica pende la autocomprensión misma de lo que es la propia filosofía.

Podemos ir ya adelantando que es precisamente en este último punto donde se encuentra el verdadero quid de nuestra cuestión. En él está el origen de las tribulaciones de Ortega con su técnica y, también, el de la precariedad de la filosofía de la técnica en el contexto del pensamiento actual. En efecto, la Meditación de la técnica orteguiana se desenvuelve admirablemente bien en lo que concierne a nuestros puntos 1 y 2. Es indudable que Ortega nos ofrece un marco conceptual lúcido, consistente y muy potente para interpretar y valorar el cambio tecnológico humano. Bastan seis afirmaciones para perfilarlo. 1) La esencia de la acción técnica humana no está en lo instrumental. 2) La esencia de la técnica hunde su raíz en el origen mismo de la inteligencia y de la condición humana definidas como permanente dialéctica entre lo que es y lo que podría ser; de hecho sólo la confrontación con lo posible permite alumbrar la noción misma de ser. 3) La capacidad de rectificar la naturaleza según designios que expresan cosmovisiones y deseos es un vector ineludible de la historia humana. 4) La evolución tecnológica de la humanidad no queda explicada con las categorías de necesidad, herramienta, adaptación o naturaleza y, por tanto, el cambio tecnológico cae realmente fuera del marco explicativo de la evolución biológica. 5) A diferentes cosmovisiones corresponden diferentes proyectos de desarrollo tecnológico y, por tanto, hay tantos vectores de progreso tecnológico como cosmovisiones humanas posibles. Y, finalmente, 6) con la Modernidad, Occidente entra en una dinámica de cambio tecnológico en la que la propia invención técnica se convierte en objeto de una profesión, la de ingeniero, y en la que la propia ciencia asume la producción técnica de ciertos fenómenos o artefactos - en condiciones reales o teóricas-como recurso explicativo fundamental ${ }^{6}$.

\section{Adán y el planeta sitibundo}

Junto con el incuestionable impulso de una síntesis genial, en la filosofía de la técnica orteguiana convergen influencias y antecedentes muy concretos, así como viejos motivos recurrentes del pensamiento de Ortega desde sus orígenes. Podríamos citar tópicos tan centrales como su convicción de que la decadencia de la España moderna procede de su activa resistencia a la modernidad; su noción de cultura humana como actividad por definición suntuaria que perfila al ser humano como un animal para quien lo necesario es lo superfluo; el

\footnotetext{
${ }^{6}$ No menos interesantes, aunque menos exploradas, son esas perspectivas de fracaso tecnológico que Ortega nos presenta en diversos momentos de su obra. Por ejemplo, la de que de pura apertura de posibilidades técnicas, de puro serlo todo posible, la historia se nos vacíe de sentido porque el poder serlo todo es, humanamente hablando, el no ser nada. O bien que los deseos se nos den ya tan hechos y estandarizados por nuestro entramado tecnológico que perdamos la capacidad de desear personalizadamente. O incluso que un entramado tecnológico traicione, por su propia lógica interna, los principios vitales que lo inspiran y, funcionando técnicamente bien, antropológicamente no funcione.

El obstinado silencio de Ortega sobre lo que parece ser una quiebra del proyecto europeo de modernidad en torno a la Segunda Guerra Mundial nos impide hoy conjeturar a cuál de estas u otras disfunciones hubiera atribuido Ortega a Auschwitz o Hiroshima, pero nótese que, así de pasada, tenemos sobre la mesa y quince años antes varias alternativas complementarias a la provocación heideggeriana o al desafuero de la racionalidad instrumental de la escuela de Frankfurt.
} 
motivo neokantiano de la cultura como aquello que no-es-naturaleza y aspira a transformar toda la naturaleza; su vindicado antidarwinismo (que no antievolucionismo) en el sentido de que la gran aventura humana no es lucha por la existencia o el sobrevivir sino por la consistencia o el vivir bien, y más que lucha contra otros es empeño junto a otros; o, finalmente, su peculiar antiprogresismo, en el sentido de que hay tantos progresos distintos como metas humanas posibles y que además el progreso no viene dado de suyo con el simple cambio histórico.

Todos estos motivos aparecen ya claramente en trabajos anteriores a 1912 -como Planeta sitibundo o Adán en el paraíso-, y en ellos se aprecian influencias sobradamente estudiadas hoy. Al revisar este inventario nos encontramos, sin duda, en la antesala de la Meditación. Pero son estos mismos motivos, tan familiares en la obra de Ortega, los que disimulan novedades esenciales en la filosofía orteguiana de la técnica, así como algunas tensiones de fondo entre ésta y el conjunto de su obra. Concretamente me parece básico insistir en tres aspectos que con frecuencia se infravaloran en la interpretación de la filosofía de la técnica orteguiana.

1. Para empezar, Ortega propone en su Meditación, y por primera vez con claridad en la historia del pensamiento -si exceptuamos tal vez a Lewis Mumford, cuya Técnica y civilización se publica en el 34-, una teoría del cambio tecnológico en la que éste no es ni determinante, ni determinado, ni neutral. Y esta línea es precisamente la que marca la división entre la filosofía de la técnica arcaica y la contemporánea. La noción positivista de que es la técnica la que determina el sentido de todo el cambio social -el Renacimiento se explica por el cañón y el Gótico por el arbotante-; la marxista clásica de que el cambio tecnológico viene determinado por los cambios en la estructura de producción -como medio de producción producido-; o las típicas "historias de inventos e inventores" que muestran el cambio tecnológico como una sucesión de invenciones "talentosas", previas a toda valoración axiológica o política y en las que se descuida tanto el uso del artefacto como la fase de innovación social, son ejemplos respectivos de nuestras tres viejas historias de la técnica ${ }^{7}$. Es precisamente el no partir de ninguno de estos tres presupuestos, como hace la Meditación, lo que inaugura la filosofía de la técnica actual. Pero es también esta misma aportación la primera en soslayarse por el Ortega más "historicista", que propende a mostrar la técnica como mera cosmovisión materializada.

2. En segundo lugar, la frase "sin la técnica el hombre no existiría ni habría existido jamás" encierra en su final una novedad filosófica tan radical que acabará asustando al propio Ortega. No hay condición humana posible al margen de o con anterioridad a la técnica. La técnica, pues, no es algo que el hombre haga con vistas a otra cosa, sino que se es humano exactamente en la medida en que se hace técnica. Esta afirmación es, desde luego, una tesis antropológica de tremendas consecuencias, pero no es sólo eso ni principalmente una tesis antropológica. Para el Ortega de la Meditación, reflexionar

\footnotetext{
7. Importa destacar que estas tres visiones "arcaicas" del cambio tecnológico promueven un modelo conceptual que imposibilita - y hace inútil- una filosofía de la técnica propiamente dicha. En la medida en que pervivan -y obviamente perviven, sobre todo fuera de la filosofía-, la filosofía de la técnica será un producto confuso, innecesario y sospechoso.
} 
o conocer un mundo es ya hacer algo desde la técnica. La razón del hombre -sea cual sea el contenido que le demos luego a este logos primordial- lo es en la medida en que se proyecta la posibilidad de otro orden de cosas sobre la facticidad de lo dado y, en este sentido, lo que enuncia la tesis de Ortega es, sobre todo, un hallazgo lógico y ontológico, es decir, de una dimensión trascendental de la razón misma, la de la tecnicidad como trascendental del ser. La inteligencia humana es siempre inteligencia creante; no hay otro modo humano de hacerse cargo de las cosas. Sólo un ser que construye casas, y precisamente porque construye casas, puede abrir los ojos y ver un bosque en una masa de árboles, y un árbol en determinado vegetal, y un vegetal en determinada cosa y una cosa, en fin, en determinada situación. Hasta la confrontación con Heidegger en los cincuenta, Ortega no volverá a recoger este motivo.

3. En tercer lugar es preciso asumir que para Ortega la técnica es, en definitiva, un suceso cósmico, y no sólo histórico o biográfico; es decir, un verdadero acontecimiento en el orden de la realidad física. El artefacto configura propiamente la materia del mundo, y por lo tanto encierra en sí también riguroso conocimiento -no sólo producción, esfuerzo o quehacer. Ortega alude varias veces a esta dimensión del acto técnico hablando del "magisterio insobornable de las cosas" sobre el espíritu". Así pues, aunque en la figuración proyectiva hay una insoslayable dimensión histórica, no hay sólo historia sino artefactos que viven ya su propio ser en el cosmos. Realidades tales como la penicilina, la bomba de Hiroshima, o el gas Zyclon B9 . Y, claro está, no se puede pretender para estas cosas el mismo estatuto ontológico que para una idea, una creencia o las cuitas del joven Werther sin hacer algo de trampa, aunque sólo sea porque, como señala Popper, cada artefacto nuestro, por sí solo, refuta un montón de teorías sobre la realidad ${ }^{10}$. Y más aún en una cultura como la moderna, cuyo hito fundacional debe situarse, como Ortega explica tan bien en tantas partes, en que se empiezan a fabricar artefactos precisamente con el expreso fin de refutar cosmovisiones.

No hace falta insistir, a la vista de lo dicho, en que la Meditación es una obra con un valor intrínseco independiente del conjunto de la obra de Ortega y, en algunos aspectos destacados, confrontada con las versiones más difundidas de la misma. En ella culmina uno de los posibles desarrollos de la razón vital orteguiana, Dtal vez el más consistente aunque, la$\mathrm{P}$ entablemente, [no fuera el que más gustase al Ortega nuclear de los años treinta y primeros cuarenta $^{11}$ ¿ ¿La habríamos llegado a conocer impresa de no darse el expolio editorial al

${ }^{8 .}$ Cf. toda la lección XII de la Meditación (V. 601).

9. En este contexto, queda claro, por ejemplo, que no se puede cambiar de sitio Ensimismamiento y alteración y llevárselo, como el sillar de un viejo templo romano, para que nos haga de dintel en El hombre y la gente como si no pasara nada. Se comprende, también, que no se puede hablar, sin más, de una teoría de la razón fantástica equivalente en La deshumanización del arte y en la Meditación de la técnica.

10. “...todo invento técnico realmente nuevo falsa una teoría hasta ese momento verificada, la teoría que no lo produce, que no puede producirlo. Que no se puede hablar a distancia y que, por tanto, no puede haber teléfonos es algo constantemente verificado hasta la invención del teléfono". Sociedad abierta, universo abierto, Tecnos, Madrid, 1994, p. 41

11. Una rigurosa y penetrante vindicación de la actualidad y vigencia de la Meditación de Ortega en el sentido señalado nos la ofrece José Luis González Quirós en "La meditación de Ortega sobre la técnica y las tecnologías digitales”, Revista de Estudios Orteguianos, 12-13, 2006, pp. 95-111. 
que Ortega alude en su presentación? Podemos dudarlo; al menos en los términos en los que se publicó y habida cuenta de que en ese preciso momento Ortega está publicando -en La rebelión de las masas, por ejemplo- que la técnica moderna es esencialmente ciencia aplicada, o que su efecto inevitable es una sociedad de masas alienante que estandariza horriblemente nuestro tiempo libre y hace peligrar el vigor de las élites sociales que inexorablemente lideran el progreso social ${ }^{12}$.

Otros frentes teóricos incómodos que la Meditación le plantea a Ortega son la dificultad para habilitar criterios universales con que preferir algunos modelos de existencia-tecnología frente a otros ${ }^{13}$; la opacidad constitutiva del futuro tecnológico humano que, de pura apertura imaginativa, Ortega no tiene ni idea de a dónde puede ir a parar, y parece históricamente indiferente; o la dificultad para justificar, desde sólo estos parámetros, el sentido de una técnica de la naturaleza interior, es decir, del alma. Dificultad con la que el propio Ortega cierra la Meditación. Todos estos elementos de fuga argumental explican, por sí mismos, que tras el 39 -en realidad desde el 35-, la filosofía orteguiana de la técnica haga mutis de puntillas. Pero no para siempre.

\section{Leibniz y la fantasía como ciencia exacta}

Estamos ahora en Lisboa y en 1947. Lejos queda el Santander del 33 y su recién estrenada Universidad de Verano, donde nos permitíamos el lujo de leer con chispeante gracejo junto a la playa de La Magdalena "dictados hechos a la carrera" de "toscas y balbucientes" ideas sobre la técnica "que pueden ser de importancia". La circunstancia biográfica es muy distinta, la histórica también. Por lo pronto, cosa extraña, en ese momento de su vida, y durante unos tres meses, Ortega vaca; es decir, no tiene a la vista un encargo concreto que dirija su trabajo. Y no es menudo el dato de que cuando el Ortega escritor, conferenciante y animador cultural puede vacar de su figura pública y ensimismarse en su labor, escoja la abrumadora empresa de escribir un monumental tratado sobre las matemáticas de Descartes, la axiomática de Euclides y la lógica de Aristóteles, como anunciada y no cumplida propedéutica a los principios metafísicos leibnicianos. Y todo ello de nuevas, sin apenas reformulación de sus temas habituales, sin ingeniosas digresiones que alivien la lectura, y, sobre todo, con una voluntad de rigor inusitada que desbanca de lejos la legendaria claridad e incluso la elegancia en la escritura.

12. Todo muy lejos todavía, como vemos, del guiño al american way of life que hará en Aspen en 1949, cuando afirme que la técnica, en América, está propiciando una democratización ciudadana insospechada y sin parangón porque lo hace precisamente desde el hobby, es decir, desde el empleo del ocio y el tiempo libre, y no desde la igualación laboral, como se daba por sentado en la vieja sociología europea. Este último inciso, de genial penetración sociológica, destaca sin embargo otro motivo argumental que es especialmente embarazoso para Ortega, porque es obvio que sólo una elite fáctica promueve de hecho nuestro cambio tecnológico, con lo que nunca es "el hombre" el que proyecta la técnica, sino siempre unos pocos hombres los que la proyectan para todos, y ello apunta a una grave aporía en la doctrina de su Meditación.

${ }^{13 .}$ Cosa que Ortega, sin duda, hace; por ejemplo al considerar objetivamente decadentes a los pueblos primitivos o en su apología de la velocidad como triunfo de la inteligencia sobre dos cosas tan estúpidas como son el espacio y el tiempo. Cf. Una interpretación de la historia universal, I. (IX. 1193). A propósito de esta obra, que es precisamente la que interrumpe la IPL, es importante destacar el permanente esfuerzo de Ortega por criticar la idea de que la técnica humana quepa bajo la categoría de challenge implementada por Toynbee, esfuerzo que, sin duda, es una de las motivaciones cruciales del escrito. 
Marías contaba que las páginas que componen La idea de principio en Leibniz y la evolución de la teoría deductiva se escribieron en ochenta días de frenética labor y sin biblioteca. Que fuera alguno más y que tuviera algunos libros a mano no menoscaba el titánico esfuerzo de ahondamiento lógico y sistematización con que Ortega sorprende aquí a propios y extraños. Es un tópico mencionar, al respecto, la sombra de un Heidegger que acaba de trepar al pedestal de filósofo del siglo XX con su Ser y Tiempo bajo el brazo. La verdad es que, más que en Heidegger, en quien hace pensar la lectura de IPL es en el propio Husserl.

Ironizando, precisamente, sobre la oscura profundidad de Heidegger, Ortega señala a su regreso de Darmstadt que un pensador no puede ser sólo profundo y que tras la inmersión hay que regresar a la superficie con una perla que poner a la luz. En esta zambullida lisboeta de escafandrista celtibérico Ortega casi se nos ahoga y, leída la obra por encima y en conjunto, no aporta ni una pequeña concha que enseñar en la playa ${ }^{14}$. Quiere la historia que pasados esos tres meses lejos de cualquier Weimar, un Weimar nuevo, venido a menos, pero Weimar al fin, vuelva a estar disponible en Madrid como Instituto de Humanidades, y se ponga así un abrupto final al descomunal trabajo iniciado. Sabemos que la obra queda cortada in fieri y que ni siquiera se completa la primera de las tres partes previstas que son, además de la IPL, El principio de la razón suficiente y El principio de lo mejor.

De entre las pocas pistas que tenemos sobre el sentido de este proyecto debemos quedarnos ahora con una referencia capital que encontramos en El mito del hombre allende la técnica, la conferencia de Darmstadt del 51, donde leemos:

"Hemos ido a caer en las más profundas honduras de la filosofía, sin habérnoslo propuesto, porque tal vez el enigma más profundo de la filosofía se encuentre en la relación entre posibilidad y realidad, como nos ha enseñado el inmortal Leibniz"15.

Aunque, desde algunos puntos de vista, el texto de esta conferencia no concuerda exactamente ni con la Meditación ni con la IPL, Ortega nos muestra aquí de nuevo, y hablando precisamente de la técnica, una motivación clave en la IPL. La motivación es patente cuando se comprueban unas pocas fechas: Ortega vuelve a su técnica en la medida en que se siente impelido a distanciarse de Heidegger. Y para ello emprende un ingente tratado sobre la relación entre posibilidad, necesidad y efectividad; es decir, sobre la modalidad del ser. Aquí está el hilo de Ariadna que nos abre el proyecto argumental sin cuya realización, según el propio Ortega, la doctrina de la razón vital no quedaría debidamente fundamentada.

"En 1925 proyectaba yo una serie de publicaciones en que me proponía formal y titularmente el "replanteamiento del problema del ser" e invitaba a mis discípulos

\footnotetext{
14. La reseña de Gustavo Bueno a la IPL aparecida en la Revista de Filosofía ( N $^{\circ}$ 68) de 1959 es paradigmática en este sentido. Con todo, parece sólida la interpretación tradicional de la IPL como un esfuerzo por mostrar que todo discurso racional se apoya de manera explícita o implícita en creencias y que el único primer principio metafísico riguroso -también en el sentido más lógico-formal de la argumentación filosófica- es el vivir propio. No es intención de este trabajo cuestionar esta interpretación general, que parece acertada en su generalidad, ni, mucho menos, agotar la relación entre Ortega y Leibniz, que desborda el marco de esta obra y que, probablemente, encuentra en las nociones de mónada y de sustancia activa de éste último unos nexos argumentales más básicos que los de la principialidad.

15. El mito del hombre allende la técnica (VI. 814).
} 
a que organizasen sus programas universitarios en función rigurosa de este concepto. (...)

En 1925 yo enunciaba mi tema -algunos de mis discípulos podrán recordarlodiciendo literalmente: $1^{\circ}$, hay que renovar desde sus raíces el problema tradicional del Ser; $2^{\circ}$, esto hay que hacerlo con el método fenomenológico en tanto y sólo en tanto este significa un pensar sintético o intuitivo y no meramente conceptual abstracto como es el pensar lógico tradicional; $3^{\circ}$, pero es preciso integrar el método fenomenológico, proporcionándole una dimensión de pensar sistemático, que, como es sabido, no posee; $4^{\circ}$, y último, para que sea posible un pensar feno P enológico sistemático hay que partir de un fenómeno que sea él por sí sistema. Este fenómeno sistemático es la vida humana y de su intuición y análisis hay que partir"16.

La declaración de la $I P L$ tiene, para el lector habitual de Ortega, un matiz dramático que no se puede soslayar. Pocas veces, tal vez ninguna, ha puesto Ortega tan claramente a la vista sus cartas ante el lector. El Ortega de la $I P L$ se nos presenta urgido por su propia trayectoria profesional a tratar de cierto tema todavía no trabajado en su producción pero ineludible: el de la relación entre ser posible y ser efectivo, a los efectos de fundamentar debidamente una doctrina de la razón vital que no siente, sin ello, debidamente fundada ni perfilada frente a Heidegger y la fenomenología.

Sin embargo, a partir de esta declaración, el sentido de la argumentación de la IPL deja de ser explícito y tenemos que jugar a adivinar adónde quería ir a parar el autor. O, incluso, adónde no quería ir e iba sin querer, porque la $I P L$ no es solamente una obra interrumpida; es, ante todo, una obra fallida. Asumida por el lector la sugerencia metodológica de abordar la cuestión en espiral, como en el sitio de Jericó, que el propio Ortega nos hace, al final no sólo se levanta el sitio sino que se olvida decir qué estábamos sitiando. Importa, pues, destacar que en este mismo momento entramos en el terreno de la ficción argumental y la conjetura razonable. ¿Qué vínculo crucial vislumbra Ortega entre la modalidad ontológica, los principios de la metafísica leibniciana y la doctrina de la razón vital?

Nuestra propuesta de trabajo ahora es simple. Vamos a sustituir la penúltima palabra de la larga cita que precede y allí donde dice: este fenómeno sistemático es la vida humana leeremos: este fenómeno sistemático es la técnica humana. Con esta maniobra se iluminan algunos niveles de comprensión de la IPL que, ciertamente, merece la pena explorar.

El primer nivel de lectura se refiere al problema de la modalidad como marco lógico ineludible de la propia ficcionalidad humana. La inteligencia fantástica, en su función primordial de soñar y pergeñar mundos posibles, asume ya la restricción lógica de lo composible -tanto dentro de cualquier mundo efectivo como en el juego de los diferentes mundos posibles- como límite intrínseco del proyectar humano. Es decir, justamente eso que Freud desactiva en el ello al colocar su capacidad de deseo más allá del principio de realidad. Lo que hace pensable este mundo es que otros mundos son imaginables, Dpero lo que hace imD ginables a esos otros mundos es que comparten una ontología fundamental con éste. [Según

16. IPL, Parag. 29 (IX. 1119). 
la célebre propuesta de Kripke esta ontología común debe respetar, al menos, el principio de contradicción y el de tercio excluso en algún nivel del discurso. Pero no basta con eso para dar cuenta de la efectuación de un estado de cosas preciso entre otros igualmente posibles. Una ontología capaz de explicar todos los mundos posibles no explica nunca realmente "este" mundo concreto - ese era precisamente el problema del materialismo, para Bergson, que explicaba cualquier universo posible ${ }^{17}$. De ahí la reiterada preocupación orteguiana por prevenirnos contra esa creencia que desactiva todas las demás y bloquea cualquier forma tanto de racionalidad como de deseo: la creencia de que todo es posible. Lo que se esconde tras esta motivación orteguiana no es trivial, un universo idiota no puede alumbrar sentido alguno para la acción humana -el gran descubrimiento del siglo XIX-pero una facticidad pura tampoco.

Un segundo nivel de lectura que aporta la consideración tecnológica de la IPL radica, precisamente, en el optimismo orteguiano. Optimismo tecnológico y, desde éste, optimismo ontológico. Incluso en el destierro lisboeta, incluso sin libros ni alumnos, incluso en la Europa del año 47, precisamente en la Europa del año 47, La IPL se concibe como una formidable declaración de optimismo ontológico-trascendental. Lo que Ortega explora en ella, en definitiva, es cómo el sentido del principio de identidad se fundamenta en el del principio de razón suficiente, y éste, a su vez, en el del principio de lo mejor. Lectura que discurre en una dirección exactamente contraria a la interpretación que Bertrand Russell propone en su Exposición crítica de la filosofía de Leibniz del año 1900. Volveremos en breve sobre la importancia de esta confrontación.

Un tercer nivel de lectura, en fin, se refiere a la naturaleza misma de la argumentación que Ortega desarrolla en la IPL. Si alguna característica novedosa está patente en lo poco que tenemos de $I P L$ esta es, claramente, su declarada vocación trascendental. En efecto, para deberle tanto al neokantismo, la figura de Kant es, posiblemente, la que más brilla por su ausencia en el conjunto de la obra de Ortega. La IPL supone un cambio fundamental -aunque no explícito- en esta dirección. Así, por ejemplo, si en 1930 Ortega se esforzaba en subrayar que los conceptos básicos de su razón vital son "ocasionales" y no trascendentales $^{18}$; el Ortega de la $I P L$ abandona por completo la contingencia de un decurso biográfico, histórico o biológico personalizado y aborda la estructura intrínseca de la racionalidad humana desde una metafísica de la ejecutividad que es, de nuevo, la que funcionaba en la Meditación. Por eso mismo, la modalidad ya no se refiere primordialmente al ser humano, o al del ser para mí, o a la estructura del ser en la conciencia, sino que se vuelve al ser como predicado universal, al ser de autores como Escoto, Suárez y Arriaga, que Ortega desem-

17. La lógica y la teoría literaria actual han caminado ya mucho por esta senda y nos muestran hasta qué punto también lo deseable, lo creíble o imaginable, por fantástico que sea, se nutre siempre de una red de posibilidades y composibilidades lógicas cuya "determinación” final es crucial para alumbrar sentido en cualquier relato humano. Lavocat, F. (ed.) La théorie des mondes possibles et l'analyse littéraire, CNRS, 2008.

18. "De ahí que los conceptos raciovitales sean de tipo ocasional. Así todas las vidas lo son porque, en cada una, la vida significa una realidad distinta: distinta aun cuando sus atributos hasta el último fuesen idénticos. Nos impondría pues la inversión del principium identitatis indiscernibilium en un principium de discerniendo idéntico". (VII.198) Publicado por primera vez por Paulino Garagorri con el título ¿Qué es conocimiento? el interesante curso del año 29 La vida como ejecución. El ser ejecutivo. Se contiene hoy como obra independiente en el tomo VIII obras completas referidas Es precisamente de este curso, a nuestro juicio fundamental para trazar el hilo de nuestro Ortega paralelo, de donde tomamos la expresión ejecutividad. 
polva en su IPL. Querer hablar propiamente del ser hablando sólo desde el Dasein, nos dice Ortega, es, sencillamente, hacer trampa ${ }^{19}$.

Ortega concibe, pues, su proyecto filosófico final como un análisis fenomenológicotrascendental de la ejecutividad como fundamento de la razón de todo ser, y la ejecutividad es siempre cosa de dos, o mejor dicho, de uno entre cosas y con cosas, es decir, de verdaderos campos pragmáticos ${ }^{20}$. Por eso le importa tanto a Ortega destacar que no es verdad que la pregunta por el ser surja cuando nos fallan las interacciones pragmáticas con las cosas, y por eso también Ortega cobra plena conciencia de esta divergencia radical con Heidegger precisamente recuperando su doctrina sobre la técnica ${ }^{21}$. No se puede despachar el ser de quien se pregunta por el ser del martillo, por un lado, y el de los martillos por otro, porque la pregunta clave sigue remitiéndose a un ser y previo al sein-martillo y al Dasein-carpintero. Es preciso denunciar, por tanto, el mito del Dasein allende el martillo.

\section{Tecnología y entendimiento agente: el logos de las cosas del mundo}

Se percibe ahora con claridad que en la IPL se está buscando, de hecho, una ruptura radical con las metafísicas de la facticidad. Pero, claro está, llevar a cabo esta ruptura no es tan sencillo. Ortega se debate ante un dilema inherente a su propio pensamiento. Por un lado, quiere mostrarnos la vida como contingencia que no rinde pleitesía a ningún entramado previo de racionalidad lógica u ontológica. El hombre es un ser naturalmente imposible pero efectivamente real, y dar razón de este factum primordial no es la cuestión en absoluto ${ }^{22}$.

Pero en este mismo sentido hallamos de nuevo en la IPL otra clave que reviste un especial interés. En un momento crucial de su argumentación Ortega incluye una amplia digresión en la que expresa su convicción de que el entendimiento pasivo aristotélico es en definitiva y en rigor la imaginación. Aunque el contexto inmediato de la observación es, claramente, una crítica al platonismo y a Husserl, se impone de nuevo la conexión con la función primordial de su razón fantástica ${ }^{23}$. Queda así sobre la mesa otra pregunta que Ortega no llega a formular expresamente pero que sobrevuela desde este momento toda su argumentación: ¿y el entendimiento agente, entonces, cuál es? Es decir: ¿cuál es el logos del mundo?

Está claro que en la obra de Ortega la naturaleza no puede obrar ya como fundamento de la técnica. Pero la razón vital es ejecutividad de cosa entre cosas, y "el mundo" que configura esta ejecutividad debe tener alguna estructura propia por mínima que sea. De ahí que la ex-

19. I.L.P. Parag. 29. (IX.1121).

20. Tal es precisamente el título y el tema del último de los cuatro textos que conforman En torno al coloquio de Darmstadt en la edición de Garagorri, en Meditación de la técnica y otros ensayos sobre ciencia y filosofía, Revista de Occidente en Alianza Editorial, Madrid, 1982.

21. I.P.L, Parag. 29, (IX. 1122).

22. Cf. Del optimismo en Leibniz, de 1947, (VI. 509). Creo que esta especie de epojé vital no refleja en absoluto ni el planteamiento ni la intención de IPL ni permite justificar ese magisterio insobornable de las cosas que haría de su ejecutividad algo radicalmente distinto del Dasein. Aunque Ortega propone en momentos como éste esta aproximación al principio de razón suficiente, es evidente que, desde ella, no se puede desmarcar del planteamiento heideggeriano ni apelar a ningún logos intrínseco a las posibilidades del mundo, un logos que es necesario para fundamentar tanto la limitación de posibilidades existenciales - sin la cual no hay verdadera capacidad de desear-, como la efectividad del artefacto humano como objeto realmente cósmico.

23. I.P.L, parag. 18, (IX.1022) 
tensa nueva nota sobre Husserl del parágrafo 18 se vertebre sobre esta declaración: “... hay que resolverse, por fin, a hacer algo que, aunque parezca increíble, nunca se ha hecho y es preguntarse perentoriamente por qué hay en el Universo algo - sea lo que sea-que nos obligue a llamarlo inteligible; por tanto en qué consiste la inteligibilidad de lo inteligible, a parte rei" 24 .

En muy pocos lugares de su obra Ortega está tan lejos de Heidegger y es, precisamente, en este punto, donde la acción técnica humana se convierte en el nuevo motivo crucial. Así, en el contexto de la técnica orteguiana, el mundo aparece ahora como una verdadera estructura en sí mismo. Y ha de ser necesaria y propiamente estructura porque es realidad radicalmente abierta a la proyección humana pero también, al mismo tiempo, realidad en la que no todo es posible de cualquier manera. Sin ello el fantasma poderoso de Nietzsche y el poeta superhombre que configura su propio universo con su propio lenguaje ronda demasiado cerca ${ }^{25}$. Ese mundo que es siempre conmigo y yo en él contiene artefactos cuya eficacia y esencia incluye, entre otras cosas, una kénosis de cualquier yo, un estar y funcionar por sí mismos. Justamente por eso, la doctrina orteguiana de la razón humana, tal y como se expresa en su Meditación, no cierra sólo el recurso explicativo a una naturaleza como instancia de sentido, sino que cierra también todo ese repertorio floreciente de metafísicas de la facticidad que ensaya el siglo XX. El mundo exige siempre una naturaleza propia, no puede ser sólo lo que nos es dado-lo que nos es verdaderamente dado, bromea ya un Ortega joven con Miguel de Unamuno, coqueteando entonces aún con el sentimiento trágico de la vida, son "palos"26-, y tampoco es cualquier cosa se nos ocurra imaginar, temer o desear. Si la vida es la función, el mundo es la estructura.

Presenciamos, pues, de la mano de Leibniz, un dilema argumental que recorre toda la obra de Ortega y que adopta fundamentalmente la siguiente forma. Sólo hay dos formas de sobrepasar el principio de razón suficiente como expresión neta de la racionalidad moderna. La una, sugerida sin querer por el propio Leibniz en su "rectificación" del argumento ontológico; postulada también desde un punto de vista "empírico" en La cuádruple raíz del principio de razón suficiente de Schopenhauer, y referida por el propio Ortega en alguna ocasión; es la denuncia expresa del postulado subyacente según el cual aquello que es real debe ser, por ello mismo, posible ${ }^{27}$. La realidad no debe pleitesía alguna a la lógica de la posibilidad, es facticidad mera y absoluta. Desde aquí podemos ir al más "coherente" de los irracionalismos o bien hacia aproximaciones al sentido del ser que son ya -irremisiblemente en el 47- heideggerianas, le guste a Ortega o no, y que remiten alguna contingencia primordial elegir entre una nutrida carta de existencialismos contemporáneos: vivencia, vida, libertad, situación, presencia del otro, etc. Se comprende que uno de los Ortegas, el menos racio y más vita del raciovitalismo -el que se demora en Weimar-, se encuentre aquí más a gusto. Pero desde aquí se queda fuera la "ejecutividad" humana y ese entendimiento agente

24. (1.c.).

25. "Es demasiado fácil pensar. La mente en su vuelo apenas encuentra resistencia. Por eso es tan importante para el intelectual palpar objetos materiales y aprender en su trato con ellos una disciplina de contención”. Meditación, XII (V.603).

26. "Lo positivo son los palos. ¿No buscan los filósofos lo fáctico, lo dado, lo gegeben? Ahí lo tienen: ¿qué es lo que nos es dado? Palos. Ahora bien, ¿a quién le dolían menos, a D. Quijote o a Sancho?” Carta a Miguel de Unamuno del 10 de febrero de 1907. En Epistolario completo Ortega-Unamuno, El Arquero, Madrid, 1987, p.163.

27. Del optimismo en Leibniz (VI.531). 
a cuya función apunta la IPL. Es decir, la razón fantástica se queda en puro fantasear y la Meditación -y con ella la propia técnica humana- no tiene cabida. En un enfoque tal, la técnica es la última quimera - cuando no pesadilla- del espíritu.

La otra opción es avalar el sentido del principio de razón suficiente con el principio de lo mejor, es decir, justamente, por el optimismo ontológico. Lo que esta lectura de Leibniz sugiere es que si el ser no es realmente bueno -o mejorable- en sí, entonces no es racional. Claro está que este bueno tira más a "perfectible por la libertad humana" que a consumado ya en su perfección, como Ortega reprocha con verdadero enojo a Voltaire que disfrazara a su público ${ }^{28}$. Pero a donde vamos, en suma, es a que toda la estructura de la razón vital depende de que nuestra praxis imprima verdadero sentido en el mundo de las cosas y es, precisamente, desde este sentido impreso en las cosas desde donde el problema ontológico reaparece ante todo como un problema de realización de posibilidades, es decir, de modalidad.

Al buscar una ontología intrínseca a la realización de mundos posibles, Ortega asume que sólo la doctrina sugerida por primera vez en su Meditación puede atestiguar que él no es Heidegger y que su razón vital estaba ya pergeñada antes de Ser y Tiempo y en coordenadas distintas. Por eso en su IPL Ortega viene a declararnos que hasta ahora sólo ha desarrollado una parte de su razón vital, pero falta aún por desarrollar esa otra mitad que linda con el dato primordial de que el hombre trae al ser efectivo seres posibles -libros, locomotoras y mandolinas- pero no de cualquier manera posible ni por cualquier razón posible. Para justificar esta situación es preciso explorar, pues, cierta estructura trascendental que es inherente a lo dado, a lo posible y a lo efectuado. La vida, como realidad radical, no contiene sólo la facticidad de un mundo ya fabricado ante mí, sino la de un mundo fabricable y fabricado por mí sin el cual no se justifica la llamada orteguiana a salvarnos con las cosas. En definitiva, lo que se vislumbra en la $I P L$ es que la pregunta final de nuestra inteligencia filosófica -y de nuestra ontología- no es tanto qué hay sino qué puede haber.

\section{Tecnología y realidad abierta}

Llevar hasta el final su propia filosofía de la técnica le hubiera exigido a Ortega una revisión de la filosofía contemporánea, y de la suya propia, mucho más sustancial de lo previsto, y le hubiera comprometido, en suma, con una clase de argumentación metafísica que violenta de raíz esa superación de toda metafísica tradicional que parece definir el progreso de la filosofía actual. Ortega tantea este dilema en la IPL y lo rehúye en otros muchos lugares porque admitirlo sitúa su doctrina demasiado en línea con el idealismo alemán, que no en vano es la tentación final permanente del raciovitalismo y también, no lo olvidemos, la tradición metafísica que primero descubre la filosofía de la técnica y que más a gusto se encuentra en ella. Como ya afirmaba Novalis -el mismo que echaba en falta una fantasía trascendental en Fichte-: "De la imaginación productiva deben deducirse todas las facultades, todas las actividades del mundo interior y del mundo exterior" ${ }^{29}$.

28. O.c. (VI.522)

29. La cita se recoge al comienzo del Ensayo sobre el sueño de la tierra, de Gastón Bachelard, precisamente de 1947 y que, curiosamente, no cita a Ortega pero se remite a Miguel de Unamuno. Particularmente sugestivo para una filosofía de la técnica es su capítulo II: "La voluntad incisiva y las materias duras". 
De ahí el embarazo y malestar del Ortega más exotérico con su Meditación, y de ahí también el embarazo del gremio filosófico actual para con una filosofía de la técnica cuya vocación hacia la "filosofía primera" es inextirpable, así como la incapacidad de tantos enfoques filosóficos que -especialmente en Europa- tienen vetado este tipo de excursiones. Así se entiende también, mal que nos pese a los orteguianos, que el único gran pensador europeo contemporáneo que tiene franco el camino para pensar la técnica desde una ontología, es decir Heidegger, sea hoy por hoy el pensador más leído, imitado y denostado -es decir, productivo- en esta materia.

Pero entre todas las salidas pergeñadas por este conato de ontología modal que supone $I P L$ hay una que me parece particularmente interesante rescatar -a modo de digresión final- como la que podría haber sido una contribución sustancial de la obra de Ortega: la que presenta el universo como una verdadera estructura de elección. Tenemos que el mundo sólo puede ser humanamente pensado, percibido y manipulado como correlato trascendental de nuestra racionalidad ejecutiva. He aquí ese particular entendimiento agente que buscábamos. Se invierte, pues, radicalmente el sentido de la acción formal del intelecto que ahora va del sujeto proyectante a la efectividad del artefacto y no, al modo tradicional, de la forma natural, ya producida por el entendimiento agente, al entendimiento pasivo. Puede verse, así, la doctrina de la inteligencia humana en la Meditación como la contradicción más rigurosa del célebre resumen de Duns Escoto: el entendimiento pasivo es el que se hace todas las cosas y el agente el que hace todas las cosas ${ }^{30}$.

Efectivamente, el mundo suscita, determina y acoge la ejecutividad humana no sólo en su sentido tradicional de lo "agible" -terreno en el que el raciovitalismo de Ortega se encuentra tan a gusto-, sino también y sobre todo en el de lo "factible", que es donde verdaderamente se juega esta ejecutividad y donde Ortega empieza a titubear. En ese bloque indisoluble que forman, para Ortega, el yo, las cosas y el mundo, la acción productiva descubre que tanto la inteligencia humana como el propio mundo sólo son concebibles bajo la forma de estructura ontológicamente abierta a la realización de artefactos y ello de manera tal que -parafraseando al propio Kant- se puede decir que la condición de posibilidad de nuestra fabricación del mundo es la condición de posibilidad del mundo mismo. En esto consiste el quehacer más radical del radical quehacer que es la vida humana, una vida que se expresa paradigmáticamente en la acción técnica, una acción que es inherentemente optimista.

Pero este optimismo no puede fundamentarse $-\mathrm{y}$ desde luego menos que nunca en el contexto del 47-, en una constatación antropológica experimental -al estilo de Rousseau-, pues ni la naturaleza ni la historia humana lo avalan, sino que debe llegar a un nivel mucho más profundo de confrontación con La cuádruple raíz del principio de razón suficiente de Schopenhauer. La tradición de racionalidad occidental, en su prístina versión leibniciana, ha ser radicalmente reformada, pero debe salvarse su racionalidad intrínseca y esto es tanto como decir que debe hallarse una nueva fundamentación ontológico-trascendental de su optimismo distinta de la del Dios Ilustrado. Ahora bien, una vez constatada la posibilidad real de que la historia humana descarrile, y una vez desactivada la función metafísica de la naturaleza - como mero objeto del saber científico natural-, queda una vía trascendental que

\footnotetext{
${ }^{30 .}$ Comentario al De Anima, III, 5.
} 
todavía puede apelar a algo. Puede apelar a que eso que llamamos la realidad comparece como verdadero espacio de verdadera proyección, elección, ejecución y creación por parte del hombre.

La acción creadora del hombre revela, así, un mundo cuyo rasgo ontológico primordial es, ante todo, el de consistir en verdadera estructura de verdadera acción proyectiva, y en tanto que verdadera estructura de elección y producción estamos ante una realidad esencialmente abierta. Es decir, ni absolutamente necesaria - como sugiere ese spinozismo cuyo mejor antídoto, según Ortega en $I P L$, es precisamente Leibniz-, ni tampoco arbitraria porque, como también Leibniz explora mejor que nadie, aunque todo lo posible es ya a su manera real y comporta una exigencia de realidad intrínseca, no todo es realmente composible.

En la medida en que la peripecia orteguiana tenga valor ejemplar para la filosofía de la técnica contemporánea, esta triple condición del mundo como estructura de decisión, no arbitraria, y no necesaria, que define la propuesta a la que se habría enderezado la $I P L$, puede considerarse también como un verdadero prolegómeno a la metafísica del porvenir. Y sólo en este contexto se entiende la tensión y el drama de Ortega cuando como europeo y exiliado en 1947, toma la pluma para recordarle a Europa que su filosofía más sana y vigorosa, más europea en suma, debe hundir su raíz en el optimismo pragmático leibniciano.

En efecto, existen determinadas afirmaciones sobre la naturaleza de la realidad que lo que hoy llamamos ciencia no puede fundamentar en virtud de su propio método. De hecho, son afirmaciones de naturaleza metateórica que, sin embargo, involucran modelos y definiciones cruciales acerca de nociones como existencia, realidad, ser, dato, hecho, conocimiento, universo, etc. La excesiva potencia teórica y la nula o problemática falsabilidad científica de estas afirmaciones no deben llevarnos a despreciar, sin embargo, su genuina función epistemológica. Los europeos hemos pasado los últimos doscientos años más o menos convencidos de que tales afirmaciones no eran objetivamente significativas o no eran necesarias para el verdadero progreso de nuestro saber. Pero si algo deja claro nuestro presente estadio tecnológico es que los modelos de racionalidad basados en esta convicción son incapaces de explicar y orientar normativamente nuestro cambio tecnológico. El proyecto de una racionalidad pura práctica fundada exclusivamente en la autonomía del sujeto y el contrato entre sujetos, es incapaz de hacerse cargo del cambio tecnológico como vector crucial de racionalidad humana. Como, además, el contexto tecnológico del siglo XX ha desactivado categorías epistemológicas tan básicas como las de naturaleza, teoría o ciencia en su sentido tradicional básicamente consensuado desde los griegos; el saber humano se encuentra hoy en una situación en la que, como señalaba Dewey, ni la certeza puede vindicarse como un rasgo esencial del conocimiento objetivo ni la ausencia de riesgos personales o compartidos como un rasgo de racionalidad ético-social.

Entre las más fértiles y cruciales de tales afirmaciones en el pensamiento contemporáneo se encuentra, precisamente, la de que eso que llamamos realidad, universo real, o lo real en sí es, ante todo, una estructura verdaderamente abierta. Con distintos matices y registros vemos esta intuición operativa en obras tan dispares como las de Popper, Wittgenstein, Bergson o Zubiri. Posiblemente una de sus formulaciones más rotundas es la teoría de la "infradeterminación" teórica de la realidad que defiende Quine ${ }^{31}$. No se trata sólo de que

${ }^{31 .}$ Cf. Quine, W.V.O., "Dos dogmas del empirismo”, en Desde un punto de vista lógico, Ariel, Barcelona, 1962. 
con el actual nivel de nuestros conocimientos sobre el universo la interpretación teórica del mismo esté abierta -lo cual, aunque sea obvio, no deja de ser importante-; lo que se dice es que, aunque tuviésemos la más plena y perfecta noticia del universo, la totalidad teórica de los datos que podría aportar a la inteligencia humana su comprensión final seguiría abierta. $\mathrm{Y}$ es en este contexto donde la filosofía de la técnica contemporánea -la de Ortega y la de todos- se encuentra abrumada por la magnitud de la tarea que le corresponde, porque caemos en la cuenta de que la verdad última del universo - material- es la verdad última de su relación -técnica- con nosotros, y aquí es donde nuestros planes en él y con él desempeñan un papel fundamental sin el que no puede desentrañarse ninguna "verdad" que concierna objetivamente al ser. Esta sería la tercera vía entre Leibniz y Schopenhauer buscada por Ortega.

La constatación de que la realidad es una estructura abierta no es filosóficamente trivial porque de hecho no es compatible con algunas teorías metafísicas que presentan hoy la realidad de la que se hace cargo la técnica como una estructura realmente cerrada o irracional o absolutamente incognoscible en sí misma. Ninguna de estas hipótesis puede admitir, de hecho, la técnica como acción real del hombre en el mundo. Al afirmar, desde la creación técnica, que la realidad, en todos sus niveles, es una estructura abierta, el mundo se postula como un verdadero sistema en el que incluso los supuestos de una complejidad infinita, o de una ausencia de fundamento temporal resultarían compatibles con una racionalidad práctica intrínseca. La irracionalidad del mundo sólo podría fundamentarse en la refractariedad absoluta del ser a los planes y motivos del hombre. Por eso es filosóficamente crucial que el mundo sea objetivamente capaz de asumir sentido y, en definitiva, de operar el bien. No es necesario afirmar, con Leibniz, que este mundo es el mejor sistema posible para que el hombre haga la mejor técnica posible. Tal vez nuestro mundo no sea el más diseñable de los mundos $-o$ tal vez sí-, pero desde luego es un mundo lo suficientemente diseñable como para que la humanidad aspire a redimirse -si no sólo, al menos también-por la clase de técnica que hace en él.

Por ello, y por cerrar, de la mano de Ortega, la analogía con la clásica argumentación kantiana, podríamos decir ahora que el cometido último de la filosofía de la técnica en nuestra nueva situación tecnocultural es el de custodiar la apertura del ser al bien. Una apertura epistemológica, ontológica y moral que sólo es posible comprender y valorar adecuadamente desde la propia filosofía de la técnica. Lo que nuestra técnica enseña al filósofo hoy es que ya no podemos hablar del ser humano hablando sólo del ser humano ni del universo hablando sólo del universo. En tanto el ser humano es, ante todo, un verdadero hacedor de mundos posibles tenemos que habilitar de nuevo un acceso al ser en todas sus acepciones modales. En realidad, nada de esto es tan extraño si reparamos y admitimos que sin la técnica el hombre no existiría ni habría existido jamás. Así, ni más ni menos. Porque el hombre es el animal de la razón y la razón es la imaginación que funciona. 


\section{Sobre el autor}

Ignacio Quintanilla Navarro: Nacido en 1960, doctor en filosofía y licenciado en psicología industrial ha impartido docencia en la Universidad Complutense de Madrid y el la Universidad Nacional de Educación a Distancia y actualmente es colaborador habitual de la Escuela de Filosofía de Madrid. Sus áreas de investigación habituales son teoría del conocimiento, historia de la filosofía moderna y contemporánea y filosofía de la técnica. En todas ellas ha publicado diversos artículos y monografías. La última es Techné, la filosofía y el sentido de la técnica, Common Ground Publishing, 2012. 